\title{
ESTUDO PRELIMINAR SOBRE A ATUAÇÃO DA ÁREA DE RECURSOS HUMANOS COMO CONSULTORIA INTERNA E SUA CAPACIDADE DE INOVAÇÃO DAS PRÁTICAS DE GESTÃO
}

\author{
PRELIMINARY STUDY ABOUT THE CAPACITY FOR \\ INNOVATION MANAGEMENT PRACTICES AND THE ROLE OF \\ HUMAN RESOURCES INTERNAL CONSULTING
}

Data de submissão: 06-10-2012 Aceite: 13-11-2014

Luciana Campos Lima ${ }^{1}$ André Luiz Fischer ${ }^{2}$

\section{RESUMO}

O presente estudo tem como objetivo verificar se as empresas que optaram por adotar em suas Áreas de Recursos Humanos (ARH) o modelo de consultoria interna inovam mais em suas práticas de gestão, do que as que possuem uma atuação tradicional. A investigação de natureza quantitativa e exploratória utilizou dados secundários pertencentes a um universo constituído por 541 empresas nacionais e multinacionais de diferentes portes, e 139 mil profissionais participantes de uma pesquisa longitudinal entre os anos de 2007 a 2011. A amostra não probabilística e típica foi composta por 26 empresas, e os achados demonstram que as ARH que adotam a consultoria interna possuíam 64 práticas em 2007 e 128 em 2011, enquanto que as tradicionais saíram de 59 práticas em 2007 para 127 em 2011 . Percebe-se que 7\% das empresas que adotam o modelo de consultoria interna não possuem um aumento constante das práticas ao longo dos anos. Já $14 \%$ das ARH que adotaram o modelo tradicional não obtiveram um aumento progressivo das práticas. Também se verificou que a ARH com consultoria interna inovou pouco mais (128 práticas), em termos de aumento de práticas do que a tradicional (127 práticas). Entretanto esse resultado não pode ser considerado significativo estatisticamente. Assim, sugere-se uma agenda de pesquisa para o aprofundamento nos temas, e o redesenho do modelo conceitual proposto.

Palavras-Chave: Consultoria Interna, Recursos Humanos, Inovação.

1 Possui graduação em Psicologia pela Universidade Presbiteriana Mackenzie, MACKENZIE, mestrado em Administração pela Fundação Getúlio Vargas, FGV e doutorado em Administração pela Faculdade de Economia, Administração e Contabilidade - USP, FEA/USP. São Paulo. São Paulo. Brasil. E-mail: lucamposlima@hotmail.com

2 Possui graduação em Ciências Políticas e Sociais pela Fundação Escola de Sociologia e Política de São Paulo (FESPSP), mestrado em Ciências Sociais pela Pontifícia Universidade Católica de São Paulo (PUC-SP), doutorado em administração pela Faculdade de Economia Administração e Contabilidade da Universidade de São Paulo (FEA/USP). Atualmente é professor no Departamento de Administração da FEA USP. E-mail: afischer@usp.br 


\section{ABSTRACT}

The proposal of the present study is to verify if companies that adopted the model of human resources internal consulting innovated more in their management practices than those who keep a traditional practice. It was included the development of a conceptual model to guide the methodology. The exploratory research used secondary data belonging to a universe of 541 national and multinational companies of different sizes, and 139000 interviwed in a longitudinal study between the years 2007 to 2011. The sample is not probabilistic and typically consisted of 26 companies, and the study's findings suggest that, the ARH that adopt the internal consulting practices had 64 in 2007 and 128 in 2011, while the traditional left of 59 practices in 2007 to 127 in 2011. Realize that 7\% of companies that adopt the model of internal consulting lack a steady increase of practices over the years. Already $14 \%$ of ARH that adopted the traditional model did not obtain a progressive practices. It was also observed that the ARH innovated with little internal consulting (128 practices) in terms of increased practical than traditional (127 practices). However, this result can not be considered statistically significant. Despite the limitations of this study, one of its contributions is regarded to a possible agenda of research.

Keywords: Internal Consulting, Human Resources, Innovation.

\section{INTRODUÇÃO}

A economia globalizada, na década de 90, oportunizou às organizações a competição em nível internacional (HARRIS, MORGAN, 1996) por meio de otimização logística, avanços tecnológicos e desenvolvimento de sistemas internacionais de normatizações (FRIEDMAN, 2007). Esse contexto gerou implicações significantes para a gestão dos recursos humanos (LAWLER III, MOHRMAN, 2003), tais como a demanda de reposicionamento da referida área como um parceiro estratégico (ULRICH, 1998).

Esse reposicionamento sugere uma capacidade adaptativa diferenciada que implica inovar em suas estruturas, processos e práticas. A Pesquisa americana SHRM (2011) constatou como foco das ações implementadas pelas empresas participantes o uso de tecnologia nas funções transacionais de RH (56\%). Já no contexto britânico a pesquisa Wers (2004) demonstrou um aumento do uso ou a adoção de testes de personalidade no processo seletivo e das práticas relacionadas a arranjos flexíveis de trabalho como homeworking e o flexitime.

Em pesquisa conduzida pela PriceWaterHouseCoopers (2002), na Europa, verificou-se o aumento da terceirização, dos serviços compartilhados e do autoatendimento, concomitantemente com o do tempo em atividades estratégicas, planejamento da remuneração e formação (3\%). Já o investimento do tempo dos profissionais de recursos humanos em atividades de folha de pagamento e benefícios reduziu em $30 \%$. Esses achados, em conjunto, demonstram um esforço em favor da atuação da área de recursos humanos como parceiro estratégico do negócio.

Os resultados das pesquisas apresentadas contribuem para a reflexão sobre transformações ocorridas na área de recursos humanos (ARH) como forma de adaptar-se às demandas contextuais, destacando-se o seu estabelecimento como parceiro estratégico, portanto, assumindo o modelo de consultoria interna. Este direciona os recursos às necessidades do negócio por meio do uso intensivo de tecnologia e da recontratação dos limites de atuação e responsabilidade com os gerentes de linha.

No que tange ao contexto brasileiro, o modelo de consultoria interna é uma prática disseminada e adotada por empresas de diferentes portes e natureza de atividade (HSM MANAGEMENT, 2006; VALOR ECONÔMICO, 2012; VOCÊ SA, 2013), apesar de não ser um tema amplamente abordado nos estudos acadêmicos. Na década de 2000, destaca-se no Brasil a busca pelo alinhamento da estratégia da ARH com o negócio via adoção do modelo de atuação como 
consultoria interna com o desafio de desenvolver políticas e práticas voltadas para à retenção de talentos (AMORIM, FISCHER, 2013). Além disso, a Pesquisa Delphi (2011) sobre tendências na $A R H$ evidenciou que os participantes consideraram o modelo de consultoria interna como a primeira posição em termos de incorporação das tendências apontadas nos anos anteriores, dessa forma, justificando a importância do tema para o campo da Gestão Estratégica de Recursos Humanos no Brasil.

Bosquetti, Parolin e Albuquerque (2009) em um estudo de caso de migração do modelo tradicional da ARH para o de consultoria interna identificaram o direcionamento dos recursos para as atividades consideradas estratégicas. Essa opção permitiu uma postura proativa da ARH diante dos clientes internos. Contudo, tais autores salientam que $(2009$, p.164) a "inovação e gestão de pessoas são dois temas amplamente discutidos na literatura, de forma isolada".

Dessa forma, o presente estudo tem como objetivo final verificar se as empresas que adotam o modelo de consultoria interna de recursos humanos inovam mais em suas práticas de gestão do que as que possuem uma atuação tradicional. Para isso, desenvolveu-se um modelo conceitual que integra as visões compreendidas no referencial teórico sobre os papeis da ARH, sua capacidade de inovação e sua relação com a vantagem competitiva organizacional. A busca pelo alcance do objetivo do presente estudo visa contribuir para a produção de conhecimento relacionado ao tema de consultoria interna da $A R H$, uma vez que sua produção acadêmica é restrita no contexto brasileiro.

\section{CONTEXTUALIZANDO O “ESTRATÉGICO” DA ÁREA DE RECURSOS HUMANOS - ARH}

O posicionamento da ARH como estratégica possui como ponto referencial em seu construto a discussão entre os modelos Michigan e do Harvard Concept de gestão de pessoas. Truss et al (1997) consideram tais modelos a partir da dicotomia da visão do homem correlacionada a teoria de McGregor, e consequentemente das estratégias de controle e direcionamento do mesmo no alcance dos resultados organizacionais.

Sob essa perspectiva, o modelo Michigan Concept ou Hard prima pelo alinhamento entre as políticas de recursos humanos, a estrutura e a estratégia organizacional, possuindo como foco o alinhamento entre as políticas de RH e estas com a estratégia. Para isso sugere que o alcance da vantagem competitiva (TICKY, FOMBRUM, DEVANNA, 1984; GUEST, 1987; STOREY, 1992) se dá por meio do uso de um controle estreito dos empregados, uma vez que esse modelo está relacionado com a visão de homem econômico da teoria X de McGregor. Já para Legge (2005) esse modelo representa um tipo de integração de fundo racionalista e normativo, uma vez que privilegia o comportamento organizacional moldado a partir de imposições externas advindas de um sistema de controle burocrático.

Já o modelo Harvard Concept ou Soft tem como premissa que o nível de comprometimento dos empregados impacta nos resultados organizacionais, assim possui como característica a aceitabilidade da responsabilidade de assegurar o alinhamento entre a estratégia competitiva e as políticas de recursos humanos por meio do exercício da liderança dos gerentes de linha. Por se basear no nível de participação e bem estar do empregado, e dessa forma, ser coerente com a visão de homem da teoria $Y$ de McGregor, prima pelo auto controle e auto regulamentação associando-se a flexibilização (BEER et al, 1984; TRUSS et al, 1997). Para Legge, (2005) ao se privilegiar o desenvolvimento do compromisso dos empregados com o negócio, proporciona-se a 
internalização dos valores organizacionais em divergência ao modelo Michigan Concept.

Armstrong (2011) recontextualiza tais contribuições teóricas e sugere um ponto de convergência entre os modelos hard e soft, ou seja, um "alinhamento flexível" baseado no atendimento das demandas oriundas do ambiente competitivo como resultado entre o alinhamento da estratégia do negócio e a adaptação constante da ARH, como forma de superar os desafios impostos pelas mudanças do contexto externo (WRIGHT, SNELL, 1998).

Tal prerrogativa propicia o advento de conceitos que remetem à gestão de recursos humanos como fonte propiciadora de vantagem competitiva, para Mabey et al (1998, pg. 25) esse é um processo de "desenvolvimento de capacidades corporativas que entregam novas estratégias organizacionais". Isso implica na emergência de dois objetivos essenciais da ARH: o primeiro relacionado ao alcance da integração vertical, ou seja, as estratégias de gestão de pessoas devem se alinhar com as do negócio; e da integração horizontal, isto é, entre as práticas que compõe as estratégias da ARH. O segundo objetivo tem suporte no provimento do senso de direção em um ambiente turbulento, possibilitando a união entre as necessidades do negócio da organização com as dos empregados.

\section{A ESTRATÉGIA DA ESCOLHA DE PAPÉIS PELA ARH}

A importância da escolha dos papéis a serem desempenhados pela ARH implica na sua atuação e consequentemente na forma como a sua contribuição ao negócio é percebida pelas diversas instâncias do âmbito organizacional. Tracey e Nathan (2002) afirmam que a ARH sofre com uma imagem negativa em decorrência de algumas circunstâncias, tais como: sua ação com enfoque na defesa dos empregados, o fato de não ser uma fonte geradora de receita; sua atuação direcionada para o apoio administrativo, e também a assumpção de um papel subserviente e reativo dentro do planejamento estratégico do negócio.

Para Armstrong (2011) durante muito tempo a ARH atuou dentro da especialidade administrativa composta pela rotina operacional, e isso fez com que historicamente não fosse reconhecida como um parceiro estratégico na maioria das organizações (BROCKBANK, 1999; LAWLER, MOHRMAN, 2000). Apesar disso, autores como De Alwis (2010), Nadarajah et al (2012) advogam que a $A R H$ vem desenvolvendo um papel diferenciado a partir de sua participação na elaboração de estratégias em conjunto com os níveis superiores de gestão do negócio.

Em 1988, Dyer e Holder recomendaram que a ARH ocupasse o papel de parceiro estratégico, sendo este composto por um conjunto articulado de ações que envolvem o desenvolvimento de alianças cooperativas com os gerentes de linha, como forma de assegurar a implementação das estratégias do negócio em tempo real; a ocupação de um espaço igualitário no planejamento e decisão estratégica e o aprimoramento da sua própria gestão.

A partir da década de 90 a literatura sobre a atuação da ARH desenvolveu-se principalmente sob a perspectiva de investimento do tempo, e da natureza das atividades de gestão de pessoas a partir da redistribuição da responsabilidade sob esta. Schuler (1990) propôs seis papéis para a ARH: gestor de negócio, designer da mudança, consultor da organização, formulador e implementador da estratégia, gestor de talentos, e gestor de ativos e controlador de custo.

A ARH, na visão de Wiley (1992), pode atuar sob uma perspectiva estratégica, e dessa forma agir como consultor, assessor, diagnosticador inovador, agente de mudança, catalisador, parceiro de negócios e/ou gerente de custo. Pode também assumir uma perspectiva relacionada aos aspectos legais e assim atuar como auditor, controller, consultor, fornecedor, e/ou conciliador. Complementa essas perspectivas a que se refere aos aspectos operacionais classificados 
como: bombeiro, defensor dos funcionários, facilitador, e/ou formulador de políticas.

Storey (1992) sugeriu uma tipologia de atuação oriunda da análise da presença de intervenções táticas ou estratégicas, originando quatro possíveis papéis: o assessor com enfoque em uma ação consultiva interna; o operador que é orientado para o atendimento dos clientes e está submetido a relação com a gerência de linha; o regulador que tem foco no acompanhamento do cumprimento das normas; e o agente de mudança que busca integrar as relações entre os empregados com as necessidades do negócio.

A escolha de papéis que auxiliem em uma atuação estratégia da ARH implica no desenvolvimento de competências e readequação de processos e práticas, portanto, a articulação entre o aprimoramento de habilidades técnicas de gestão de pessoas, e a opção por práticas e processo adequados ao negócio são críticos no reposicionamento da ARH (KESLER, 1995). O autor expande a análise dos papéis para pontos que considera fundamentais para que a ARH possa efetivamente se reposicionar estrategicamente, são eles:

- A orientação da ARH para ser realmente estratégica não deve se limitar ao estabelecimento de parceria com o cliente interno, mas sim em direção ao externo, como forma efetiva do atendimento dos objetivos do negócio

- Os líderes da ARH devem ter como competência crítica a gestão eficaz das partes interessadas, que diferem das circunstâncias de construção de consenso;

- As atividades burocráticas da gestão de pessoal devem ser revistas à luz da relação de custo e benefício para quem a usufrui, no caso, os gerentes de linha, portanto, a decisão de eliminá-la ou de distribuir sua responsabilidade entre os usuários, apesar de impopular, faz se necessário.

Beatty e Scheneier (1997) corroboram com as proposições acima, defendem que o posicionamento como parceiro de negócio, apesar de almejado, carrega um potencial problema, pelo fato da ARH se posicionar como parte do time que suporta a estratégia do negócio (ULRICH, 1998). Do ponto de vista dos autores, agregar valor vai além de satisfazer as necessidades do cliente interno, significa adicionar valor real, econômico à organização. Para isso as estratégias de recursos humanos devem focar os clientes externos e investidores, assim a ARH deveria se posicionar como mais um player.

Wilhelm (1990) reforça que a atuação estratégica da ARH e sua ascensão como parceiro do negócio, tem que ir além de um "contrato" para gerar os resultados desejado, também envolvem aspectos como liderança, planejamento estratégico e conhecimento do negócio como habilidades anteriormente não presentes, agora compreendidas como cruciais para uma nova definição de sua função.

\section{A CONSTRUÇÃO DE UMA PARCERIA ESTRATÉGICA: A ARH COMO CONSULTORIA INTERNA E SUA CONTRI- BUIÇÃO AO NEGÓCIO}

Como já discutido anteriormente, uma maior contribuição para o negócio, a partir da $\mathrm{ARH}$, depende da capacidade de redesenho de seus processos, da adoção de novas competências que suporte sua participação em nível de elaboração, implementação e decisão da estratégia, além dos novos acordos com relação aos gerentes de linha. Para Kesler (1995) tais mudanças, 
para serem duradouras, implicam no realinhamento das estruturas organizacionais de forma a concentrar os serviços administrativos. Dessa forma, direcionando a função da ARH como consultoria interna e substituindo as unidades funcionais tradicionais.

A configuração da ARH como consultoria interna consolidou-se na década de 90, como resposta competitiva às demandas geradas pelas mudanças econômicas, sociais e políticas (JOHRI, COOPER, PROKOPENKO, 1998), se originou na Europa e nos Estados Unidos em meados da década de 1950. Teve como fatores impulsionadores o controle de gastos com consultorias externas, a assumpção das funções de recursos humanos ao nível da estratégia corporativa, e as inovações em tecnologia da informação (KENTON, MOODEY, TAYLOR, 2003).

Segundo o estudo realizado por Barreto et al (2011) sobre temas emergentes em recursos humanos nas produções nacionais e internacionais, destacaram-se dois temas como principais tendências na década de 90: (i) a configuração de arquitetura da ARH com foco nos sistemas de trabalho de alto desempenho, e (ii) a execução das políticas e práticas da ARH como garantia de realização da estratégica do negócio, a partir do modelo de consultoria interna.

Ulrich vem sendo referenciado, tanto no âmbito nacional quanto internacional, como um dos estudiosos preeminentes no desenvolvimento do construto a cerca de consultoria interna e parceria estratégica da ARH com o negócio. Em 2005, Ulrich e Brochbank desenvolveram uma proposta de composição dos papéis a serem assumidos pela $A R H$, utilizando para isso dois eixos, um pertinente ao foco no curto/longo prazo e outro referente as atividades estratégicas/ operacionais ligados ao processo ou a pessoa.

Essa proposição originou quatro papeis: o de administração de estratégias de recursos humanos (parceiro estratégico), o de administração da infraestrutura da empresa (especialista administrativo), o de administração da contribuição dos funcionários (defensor dos empregados), e o de administração da transformação e da mudança (agente de mudanças).

Replicando o referido modelo em um estudo empírico, Lemmergaard (2009) teve como objetivo investigar o desempenho do papel da ARH. As evidências encontradas sugerem que não só eram as quatro funções fortemente representadas, mas que elas também eram compartilhadas entre o executivo de $\mathrm{RH}$ e os gerentes de linha. Os achados mostraram que o executivo de $\mathrm{RH}$ pode atuar como especialista administrativo e agente de mudança, simultaneamente. Além disso, que os executivos de RH viam a si mesmos como defensores dos empregados, ocasionando em alguns momentos o seu desacordo com os gerentes de linha.

Lawlwer e Mohrman (2000) realizaram dois estudos sobre a incorporação do papel de parceiro estratégico por parte da ARH, o primeiro em 1998 verificou que o discurso ainda era mais evidente do que a prática. O segundo em 2001 com 150 empresas de grande porte constatou, novamente, que as mudanças nas práticas da ARH e na forma como esta gasta seu tempo ainda estavam fortemente relacionadas as atividades tradicionais de recursos humanos. Sendo assim, insuficientes para o aumento da adição de valor ao negócio e, consequentemente, pouco percebida como um parceiro no negócio.

Em 2008, Ulrich, Jounger e Brockbank revisam a proposta inicial e avançam para a concepção da ARH como um negócio dentro do próprio negócio, sendo que sua estrutura e funcionamento deve refletir a da organização empresarial, como forma de criar valor. Assim ARH estaria circunscrita a cinco funções e responsabilidades, sendo elas: 1- centro de serviços, 2- RH corporativo, 3- parceiro de negócio, 4 - centro de expertise, 5 - executor operacional.

A atuação como parceiro do negócio, segundo os autores, envolve a representatividade dos interesses dos empregados e as implicações de mudanças; a definição dos requisitos para o alcance de metas mediante a seleção e implementação de práticas apropriadas estrategica- 
mente; e complementadas com a mensuração do desempenho dos investimentos de recursos humanos.

Em estudo sobre o papel estratégico da ARH na China entre 1999 e 2006, Sumelius et al (2009) constataram que em 2006 o papel da ARH foi percebido como mais estratégico quando comparado a 1999. Além disso, também identificaram que o tamanho tanto da subsidiária quanto da ARH foram positivamente associados a atuação estratégica da ARH.

Vosburgh (2007) descreve que a consultoria interna, como configuração de ARH, envolve a venda de serviços, de intervenções únicas e de diferentes tipos. $\mathrm{O}$ objetivo é o desenvolvimento de uma relação de longo prazo entre a ARH e o cliente interno, para isso o tipo de venda é realizado por meio do papel de assessor, que almeja a confiança. Dessa forma, fazem se necessários três aspectos: a criação de soluções de trabalho, a educação constante dos clientes e a operação com sucesso das transformações ambientais.

Em uma pesquisa realizada no setor de manufatura na Malásia sobre os fatores dificultadores da atuação da ARH como parceiro estratégico, foi evidenciada como principal dificuldade a falta de competências críticas dos profissionais de RH para aumentar a eficácia dos processos (CHOI et al, 2010). Em outro estudo conduzido por Wang e Niu (2010) com 112 empresas em Taiwan demonstraram que o papel da ARH influencia o desempenho das empresas, especialmente ao atuar como agente de mudança.

Essas constatações reforçam as proposições citadas anteriormente por Vosburgh (2007), e destacam a necessidade de desenvolvimento das competências específicas dos profissionais da ARH ao se tratar da atuação como consultoria interna para o estabelecimento da parceria estratégica com o negócio. A contribuição da área, nesse tipo de atuação, se sustenta no encontro com as necessidades dos clientes, explorando as atividades transformacionais a partir da integração entre a estratégia de gestão do capital humano com as do negócio, e principalmente tomando decisões pautadas em dados rigorosos sobre a gestão das pessoas (VOSBURGH, 2007).

No cenário brasileiro, a adoção da configuração de consultoria interna é recente, aproximadamente na década de 80, influenciada pela expansão do parque empresarial e da ascensão da relação fornecedor-cliente (OLIVEIRA, 1999). De acordo com Mancia (1997), esse tipo de forma de atuação vem se fortalecendo nas organizações em decorrência de sua contribuição nos processos de mudança organizacional, como fator de renovação interna.

Pesquisas sobre o assunto tem apontado a adoção da configuração de consultoria interna pela ARH como uma alternativa promissora no que tange a percepção de aumento do nível de satisfação dos clientes internos (MOURA, SOUZA, 2008; FREITAG, GIRARDI, 2010), e no aprimoramento do papel da ARH (FERREIRA, TEIXEIRA, 2006).

Um viés recorrente na literatura que envolve a atuação da $A R H$, como consultoria interna, é a importância do suporte que os processos e práticas de gestão fornecem à assunção desse tipo de papel. Assim, a lógica é que a inovação constante dos processos e práticas favorece a sua adequação as pressões oriundas do mercado em que o negócio está inserido.

As práticas de pessoal que surgiram com a função da gestão de recursos humanos na década de 1980 incluem a gestão por competências, gestão de desempenho, a organização de aprendizagem, gestão do conhecimento e, mais recentemente, a inteligência emocional. Para Armstrong (2000) foi essa inovação que permitiu a alteração do departamento pessoal para a gestão estratégica da ARH.

Bech e Huselid (1998) advogam que as práticas de recursos humanos influenciam no valor de mercado das empresas, uma vez que as organizações podem alavancar a excelência operacional e estratégica a partir da função e dos sistemas da ARH. Portanto, é necessário que 
esta seja ciente da demanda evolutiva do negócio e como as novas ideias e boas práticas podem sanar tais necessidades (PURCELL, 1999; ARMSTRONG, 2000).

Bosquetti, Parolin e Albuquerque (2009) em um estudo de caso de migração do modelo tradicional da ARH para a de consultoria interna identificaram práticas de terceirização de processos administrativos, como forma de direcionar os recursos para atividades consideradas como estratégicas. Essa opção permitiu uma postura proativa da ARH diante dos clientes internos, numa relação de prestação de serviços, uma vez que as inovações em políticas e práticas representaram vantagens competitivas à empresa.

\subsection{A INOVAÇÃO DAS PRÁTICAS DE RECURSOS HUMANOS (PRH) COMO SUSTENTAÇÃO DA ARH COMO CONSULTORIA INTERNA}

Para Agarwala (2003) a inovação em PRH trata-se de uma questão de sobrevivência das organizações e não mais uma tendência. Assim, podem ser definidas como novas ideias, programas, práticas ou novos sistemas adotados que estejam alinhados à função de recursos humanos (WOLFE, 1995). São também consideradas como inovações administrativas, o que no conceito de Evan (1966) e Knight (1967) trata-se de inovações organizacionais ou de pessoas.

Esse construto sobre inovação em PRH é complementado com o fato de que o propósito da inovação diz respeito a sua capacidade de influenciar ou adaptar as habilidades, comportamentos e interações dos funcionários, ao criar novas capacidades e competências (SOM, 2007). Dessa forma, em estudo realizado em 1994, com 40 empresas públicas, sobre a relação entre o ambiente externo e as inovações em PRH, Tannenbaum e Dupuree-Bruno encontraram uma forte relação entre ambos.

Os referidos autores tiveram como principais achados: i) as empresas grandes são mais adeptas a inovação em PRH do que as pequenas; ii) as organizações com maior nível de descentralização demonstraram menor nível de inovação em PRH; iii) as empresas com maior nível de formalização demonstraram maior número de inovação em PRH. Portanto, os resultados corroboram com a proposição de Som (2007), uma vez que esses achados podem suscitar reflexões sobre os aspectos da capacidade de influência da constituição das áreas nos comportamentos e na inovação.

Agarwala (2003) realizou um estudo com 422 executivos e gestores de sete organizações, obteve como resultados que $46 \%$ dos entrevistados acreditavam que as inovações em PRH foram importantes para a consecução dos objetivos dessas organizações. Porém, no que tange a percepção do grau de incorporação de tais inovações em PRH, encontrou como média $36 \%$, e com relação ao nível de satisfação com a implementação da inovação das PRH teve média de $35 \%$.

Autores como Damanpour (1991) e Wolfe (1995) afirmam que a implementação de inovações nas organizações dependem da valorização de determinados atributos, que no caso das PRH se referem a: 1- falta de conhecimento entre as entradas, processos e resultados da inovação; 2- a relevância da inovação, se técnica ou administrativa; 3- ao nível de radicalidade da inovação que implica em novos comportamento na mesma medida; 4- na magnitude da extensão da mudança, se abrange estrutura, pessoal e recursos financeiros; 5- no potencial de infiltração, ou seja, no número de pessoas abrangidas.

Parolin e Albuquerque (2011) em estudo sobre gestão estratégica de pessoas para a inovação citam a estrutura organizacional, a filosofia e valores organizacionais, as políticas e sistemas de recursos humanos como constructo, que ao serem evidenciados nas estratégias de gestão de pessoas aumentam a abrangência de obtenção de um ambiente favorável à inovação. 
Wolfe, Wright e Smart (2006) escreveram um artigo em que analisaram a implementação de inovações em PRH relacionadas à história do Moneyball, entre os achados destacam-se as questões pertinentes à superação da resistência, e como a inovação em $\mathrm{PRH}$ pode contribuir para a manutenção da vantagem competitiva. No contexto brasileiro, os estudos da inovação em PRH demonstram possuir algumas limitações, por não serem amplamente discutido. Nas palavras de Bosquetti, Parolin e Albuquerque (2009, p.164): "inovação e gestão de pessoas são dois temas amplamente discutidos na literatura, porém de forma isolada".

\section{UM MODELO INTEGRADOR COMO FORMA DE ORGA- NIZAÇÃO DA ANÁLISE DOS RESULTADOS.}

A argumentação construída ao longo do referencial teórico, que percorre as bases da gestão estratégica de recursos humanos, discute as vicissitudes dos papeis da $\mathrm{ARH}$, discorre sobre sua atuação como consultoria interna, principalmente, no seu reposicionamento como um parceiro do negócio mediante a sua capacidade de inovação, permite a construção de um modelo conceitual integrador, conforme descrito na Figura 1:

Figura 1: Modelo Conceitual Integrador da ARH como Consultoria Interna e Inovação nas PRH

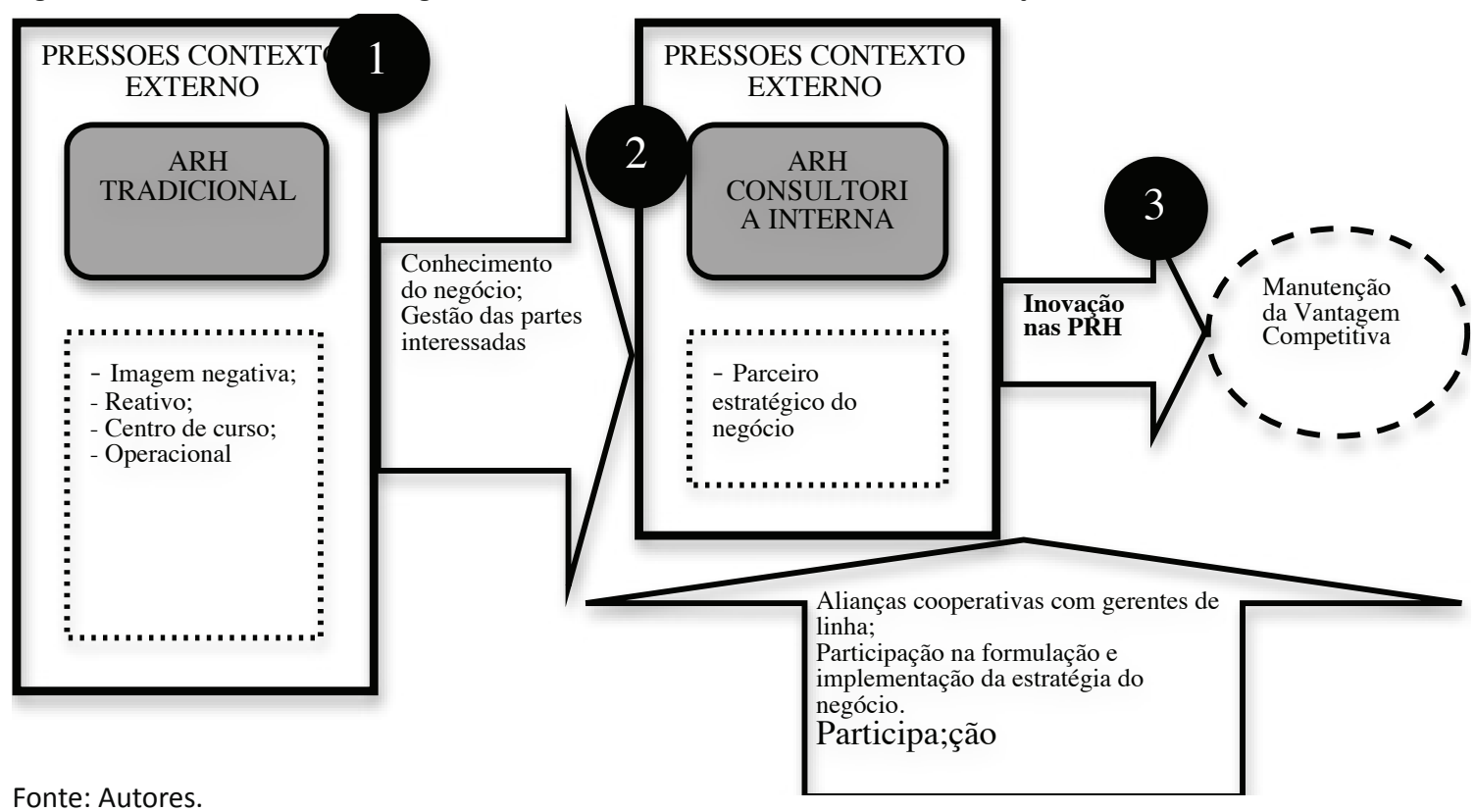

O modelo conceitual é constituído por três partes, cada uma delas subsidiada pelo referencial teórico pertinente. Assim, a primeira parte do modelo conceitual se refere ao subcapítulo três, ou seja, da ARH tradicional e de sua imagem perante o cliente interno. Já a segunda parte do modelo conceitual diz respeito ao subcapítulo quatro que trata sobre da constituição da ARH como uma consultoria interna. E finalmente, a terceira parte é oriunda do subcapítulo 4.1 que versa sobre a inovação em práticas de gestão de pessoas.

A proposta do modelo conceitual, além de integrar a construção teórica histórica no que diz respeito aos papeis da $A R H$, assume que ao se tornar uma consultoria interna, a ARH inovaria com mais frequência suas práticas de gestão do que a função tradicional de recursos humanos. Isso porque essa teria uma maior capacidade de adaptação constante às pressões do contexto externo. 
A capacidade de inovação em práticas de gestão de pessoas possui como base um perfil de competências diferenciado dos profissionais de $\mathrm{RH}$, inclusive com maior conhecimento do negócio organizacional, em busca do fortalecimento do atendimento das demandas de todas as partes interessadas e não apenas no cliente interno. Além disso, também supõe a formação de alianças como os gerentes de linha, o foco nas atividades transformacionais oriundas da participação efetiva da ARH na concepção e implementação da estratégia.

Tal modelo foi utilizado no desenvolvimento do trajeto metodológico do presente estudo, a partir da possibilidade de verificação de sua validade, circunscrita ao quesito inovação de práticas na relação ARH tradicional versus ARH consultoria interna.

\section{TRAJETO METODOLÓGICO}

A investigação de abordagem quantitativa constitui-se de natureza exploratória por proporcionar uma visão a respeito das características de um fato pouco explorado, em que não há conhecimento acumulado e sistematizado sobre o assunto (VERGARA, 2007; SELLTIZ, 1974).

Foram utilizados dados secundários pertencentes a um universo constituído por 541 empresas nacionais e multinacionais de diferentes portes, e 139 mil profissionais participantes de uma pesquisa longitudinal entre os anos de 2007 a 2011, com foco na identificação dos índices de qualidade do ambiente de trabalho e das práticas de em recursos humanos.

A amostra de conveniência e não probabilística (SELLTIZ,1974) foi constituída pelas empresas que demonstraram recorrência em seus índices (igual ou maior de 70\%) durante os anos pesquisados. A opção por esse banco de dados diz respeito ao vasto conteúdo relacionado à construção da realidade das práticas de gestão de pessoas reconhecidas como favoráveis por permanecerem com tais resultados ao longo dos anos.

Na referente pesquisa há dois tipos de coletas de informações, a primeira composta pelos empregados que participam voluntariamente respondendo a 64 questões com escala Likert de 5 pontos que investigam a sua relação com a instituição, com os gestores, e com os processos de trabalho e gestão.

A segunda fonte de coleta de dados é com os representantes das empresas, estes respondem um questionário específico sobre os seguintes os fatores - estratégia e gestão, liderança, políticas e práticas, e cidadania empresarial - acrescido do preenchimento de um caderno de evidências das práticas de gestão que foram declaradas. Foi esse banco de dados que possibilitou a realização do presente estudo.

As etapas constituintes do processo investigatório foram:

1. Identificação no ranking dos resultados longitudinais das empresas que permaneciam de 2007 a 2011 como destaques em seus resultados de gestão de clima.

2. Análise dos conteúdos dos caderno de evidências das empresas identificadas na etapa 1, para a distinção das que declararam que possuíam o modelo de consultoria interna de recursos humanos, das que atuavam tradicionalmente.

3. Análise do conteúdo dos questionários respondidos pelos representantes das empresas, para mapear as frequências das práticas de recursos humanos, verificando quantitativamente o acréscimo/exclusão/permanência de programas durante o período de 2007 a 2011. Nessa etapa foi utilizado o conceito de Wolfe (1995) sobre inovação em RH como critério de análise, ou seja, foi consideração como inovação em $\mathrm{RH}$ a adoção de novas práticas ao longo dos anos estudados. 
Tomou-se como referência na análise da inovação, o conjunto de práticas do ano de 2007. Foram excluídas as relacionadas a benefícios concedidos aos empregados, e também os oriundos dos subsistemas correlatos como a área de saúde, ou seja, o levantamento das práticas foi restrito ao escopo das funções básicas da área de recursos humanos (recrutamento, seleção, treinamento, desenvolvimento, gestão do clima, carreira e remuneração). Também não foi considerado para o presente estudo a predominância da inovação de acordo com a função, ou seja, privilegiou-se o entendimento geral da adoção das práticas.

\section{ACHADOS DO ESTUDO À LUZ DO MODELO CONCEI- TUAL INTEGRADOR}

A amostra foi composta por 26 empresas, destas não foi possível identificar qual o tipo de modelo adotado pela ARH em 9 empresas, 13 possuem a consultoria interna como atuação da ARH, e 14 com funcionamento tradicional. Como forma de constituição de um banco de mesma base, optouse por excluir, de forma aleatória, uma das empresas que adotam o modelo tradicional, ficando assim o banco formado pelo mesmo número de empresas com e sem consultoria interna (13 cada uma).

Utilizando como base o modelo conceitual integrador e seu pressuposto de que a área de recursos humanos quando configurada como consultoria interna tenderia a inovar mais do que uma área de funcionamento tradicional, segue-se a análise dos resultados a partir da apresentação da Figura 2.

Figura 2: ARH que adotam o modelo de consultoria interna $\times \mathrm{ARH}$ tradicionais

\begin{tabular}{|c|c|c|c|c|c|}
\hline $\begin{array}{c}\text { CONSULTORIA } \\
\text { INTERNA RH }\end{array}$ & $\mathbf{2 0 0 7}$ & $\mathbf{2 0 0 8}$ & $\mathbf{2 0 0 9}$ & $\mathbf{2 0 1 0}$ & $\mathbf{2 0 1 1}$ \\
\hline E1 & 9 & 7 & 8 & 9 & 9 \\
\hline E2 & 4 & 5 & 8 & 9 & 10 \\
\hline E3 & 6 & 6 & 8 & 8 & 10 \\
\hline E4 & 4 & 7 & 8 & 9 & 10 \\
\hline E5 & 5 & 6 & 8 & 9 & 10 \\
\hline E6 & 5 & 5 & 9 & 7 & 10 \\
\hline E7 & 5 & 7 & 9 & 9 & 10 \\
\hline E8 & 3 & 6 & 8 & 8 & 10 \\
\hline E9 & 4 & 6 & 8 & 8 & 10 \\
\hline E10 & 6 & 7 & 8 & 8 & 9 \\
\hline E11 & 3 & 7 & 8 & 8 & 10 \\
\hline E12 & 5 & 6 & 8 & 8 & 10 \\
\hline E13 & 5 & 6 & 7 & 8 & 10 \\
\hline Total de práticas & $\mathbf{6 4}$ & $\mathbf{8 1}$ & $\mathbf{1 0 5}$ & $\mathbf{1 0 8}$ & $\mathbf{1 2 8}$ \\
\hline
\end{tabular}




\begin{tabular}{|c|c|c|c|c|c|}
\hline RH TRADICIONAL & 2007 & 2008 & 2009 & 2010 & 2011 \\
\hline E14 & 4 & 8 & 9 & 9 & 10 \\
\hline E15 & 4 & 6 & 9 & 8 & 10 \\
\hline E16 & 5 & 7 & 8 & 8 & 10 \\
\hline E17 & 5 & 7 & 8 & 8 & 10 \\
\hline E18 & 4 & 6 & 8 & 8 & 10 \\
\hline E19 & 4 & 7 & 8 & 9 & 7 \\
\hline E20 & 5 & 6 & 8 & 8 & 10 \\
\hline E21 & 5 & 6 & 8 & 8 & 10 \\
\hline E22 & 4 & 6 & 9 & 9 & 10 \\
\hline E23 & 4 & 3 & 8 & 9 & 10 \\
\hline E24 & 5 & 7 & 8 & 8 & 10 \\
\hline E25 & 4 & 7 & 8 & 8 & 9 \\
\hline E26 & 6 & 4 & 7 & 9 & 11 \\
\hline Total de práticas & 59 & 80 & 106 & 109 & 127 \\
\hline
\end{tabular}

Fonte: Autores.

Os achados, sugerem a validação da afirmação de Agarwala (2003) sobre a inovação em PRH ser considerada não mais como uma tendência, mas como uma forma de sobrevivência, não apenas da área de recursos humanos, mas também das organizações. Isso porque, independente do tipo de modelo adotado pela ARH evidenciou-se o crescimento constante da inovação em suas práticas de gestão de pessoas entre os anos de 2007 e 2011.

As que adotam a consultoria interna possuíam 64 práticas em 2007 e 128 em 2011, enquanto que as ARH tradicionais saíram de 59 práticas em 2007 para 127 em 2011. Percebe-se que $7 \%$ das empresas que adotam o modelo de consultoria interna não possuem um aumento incremental das práticas ao longo dos anos.

Já as ARH que adotaram o modelo tradicional não obtiveram um aumento progressivo de práticas (15\%). Tais achados estão em concordância com o modelo conceitual integrador, uma vez que sua premissa é que as empresas que adotam a consultoria interna como estratégia de atuação possuem uma capacidade de inovar maior do que as de gestão tradicional na ARH.

Também se verificou que a ARH com consultoria interna inovou mais (128 práticas), em termos de aumento de práticas do que a tradicional (127 práticas). Apesar disso, a diferença entre o volume de práticas não pode ser considerado significativo estatisticamente. Um ponto a ser considerado é a oscilação entre o volume de praticas concentrou-se entre os anos de 2007 e 2008, enquanto que a partir de 2009 o comportamento das práticas pareceu mais similar em ambos os modelos.

A análise dos dados traz à tona a questão do comportamento das práticas, ou seja, inovar pode ser compreendido como uma estratégia, da mesma forma que a exclusão de práticas. Esse viés de interpretação dos dados parece conectar-se com o conceito de alinhamento flexível de Armstrong (2011). Isso porque, em determinados momentos, as demandas externas a organização e as solicitações de articulações entre práticas no contexto interno sugerem uma adequação de práticas que não necessariamente signifique a inovação em gestão de pessoas.

Deve se considerar também que a amostra da pesquisa é composta por empresas que primam pela qualidade dos resultados da gestão de clima, podendo ter como fator significante de melhoria do ambiente de trabalho a implementação constante de novos programas, sem considerar para isso o modelo adotado pela ARH. 
O fato das ARHs terem se destacado igualmente nos resultados da investigação, podem ser consideradas à luz dos resultados da pesquisa realizada por Lawlwer e Mohrman (2000) citada anteriormente. Ou seja, é possível que atuação da ARH, como consultoria interna, ainda seja constituída fortemente por um discurso que não se sustenta na realidade cotidiana das organizações.

Outra questão tem a ver com os fatores estimuladores ou restritivos descritos por Damanpour (1991) e Wolfe (1995), sobre o processo de inovação. A partir dos achados citados supõe-se que a partir de 2009 fatores como a falta de conhecimento entre as entradas, processos e resultados da inovação; ou mesmo relacionados com a relevância e magnitude da inovação possam ter sido conduzidos de maneira favorável ao comportamento das práticas de gestão. Portanto, favorecendo o seu incremento.

Tal suposição remete a necessidade de se compreender a forma como a consultoria interna de recursos humanos está sendo constituída. Em que medida, a realidade organizacional está sendo alicerçada nos construtor descritos no referencial teórico? Esse aprofundamento permitirá uma avaliação complementar aos dados apresentados como resultados.

\section{CONSIDERAÇÕES FINAIS}

O presente estudo teve como objetivo final verificar se as empresas que optam por adotar o modelo de consultoria interna de recursos humanos inovam mais em suas práticas de gestão do que as que possuem uma atuação tradicional. Para auxiliar no trajeto metodológico foi desenvolvido um modelo conceitual, que integrou as diversas abordagens teóricas sobre a atuação da ARH como consultoria interna, e as proposições que essa opção estratégica confere em termos de manutenção da vantagem competitiva da empresa e o que a diferencia do modelo tradicional de área.

Retomando ao modelo conceitual integrador, pode-se afirmar que, diante dos achados do estudo, as ARH que adotam o modelo de consultoria interna inovam pouco mais em suas práticas de gestão de pessoas do que as que possuem o modelo tradicional. Entretanto por ser um estudo exploratório, com uso de técnica estatística simples, não é possível realizar qualquer tipo de generalização, pelo contrário recomenda-se o desenvolvimento de estudos mais aprofundados sobre o tema.

Além disso, a análise mais detalhada verificou que tão importante quando a constatação da inovação nas práticas de gestão de pessoas é o estudo do seu comportamento, uma vez que se percebeu a existência da exclusão de práticas ao longo dos anos, e que poderia ser um indicativo da proposição de Armstrong (2011) sobre alinhamento flexível da ARH.

O estudo da inovação em práticas de gestão de pessoas, ainda é bastante restrito, assim buscou-se nessa pesquisa, um primeiro contato e aproximação entre esses dois focos de investigação, fazendo uso para isso, de um outro conceito também pouco explorado academicamente no contexto brasileiro: consultoria interna de recursos humanos.

Dessa forma, apesar das limitações que esse estudo possui, uma de suas principais contribuições diz respeito a uma possível agenda de pesquisa sobre o tema, compreendendo:

- Uma análise detalhada de como estão estruturadas as consultorias internas das empresas estudadas, se estão alinhadas com os conceitos tratados no referencial teórico do estudo, uma vez que a identificação da existência da consultoria interna foi baseada na declaração realizada nos relatórios;

- Um estudo profundo sobre as práticas que foram inovadas, se tratam efetivamente de algo que não existia no contexto organizacional, ou se representam um aperfeiçoamento das existentes; 
- Verificação das práticas que foram excluídas, das razões, motivos e dos impactos de sua ausência;

- As diferenças dos resultados de gestão de clima e posicionamento no mercado das empresas que possuem a consultoria interna e das que não possuem.

As respostas referentes aos questionamentos acima citados possibilitaria a complementação do entendimento do modelo conceitual integrado, e colaboraria para a ampliação dos achados nesse campo de conhecimento, que aparentemente, se desenvolveu mais nos âmbitos comerciais e profissionais do que no universo acadêmico.

\section{REFERENCIAL}

AGARWALA, T. Innovative Human Resouce practice and organizational commitment: an empirical investigaton. In The International Journal of Human Resource Management, 14:2, 175-197, 2003.

ALBUQUERQUE, L. G., BOSQUeTtI, M. A., PAROLIM, S. R. H. - A Integração Estratégica entre Inovação e Gestão de Pessoas. In: Gestão de Pessoas - Perspectivas e Estratégias. (Org.) AlbuQUerque, L. G., LEITE, N. P., Editora Atlas, São Paulo, 2009.

ARMSTRONG, M. Handbook of strategic human resource management, $5^{\text {th }}$ edition, koganPage, 2011.

AMORIM, W.A.C.; FISCHER, A.L. Relações de Trabalho, Administração de Recursos Humanos e Ambiente Econômico e Social no Brasil: uma Visão Geral Sobre o Período 1990-2012. Temas de Economia Aplicada Fipe, 2013.

BARRETO, L.M.T.S.; SILVA, M.P.; FISCHER, A.L.; ALBUQUERQUE, L.G.; AMORIM, W.A.C. Temas emergentes em gestão de pessoas: uma análise da produção acadêmica In Rev. Adm. UFSM, Santa Maria, v. 4, n.1, p. 215-232mai./ ago. 2011.

BEATTY, R.W.; SCHNEIER, C.E. New HR roles to impact organizational performance: from "partners" to "players". Human Resource Management, 36(1), 29-37, 1997.

BEER, M; SPECTOR, B; LAWRENCE, P.; MILLS,
D; WALTON,R. Managing human assets. New York: Free Press, 1984.

BROCKBANK, W. If HR were really strategically proactive: Present and future directions in HR's contribution to competitive advantage. In Human Resource Management, 38, 337-352, 1999.

CHOI, S.L; WAN KHAIRUZZAMAN, W. I.; SALMIAH, M. A. An exploratory study on the Malaysian human resource professionals in the manufacturing sector. In International Journal of Business and Society, 11 (1), 89-105, 2010.

DAMANPOUR, F. Organizational innovation: A meta-analysis of effects of determinants and moderators. Academy of Management Journal, 34, 555-590, 1991.

DE ALWIS, A. C. The impact of electronic human resource management on the role of human resource managers. In Economic a Management, 4, 47-60, 2010.

DELPHI RH: Tendências em Gestão de Pessoas nas Empresas Brasileiras- Edição Confirmatória, 2011.

DEVANNA, M.A.; FOMBRUN, C; TICHY, N. A framework for strategic Humam Resource Management. In FROMBUN, C; TICHY, N.M.; DEVANNA, M.A., Strategic Humam Resouces Management. New York; Wiley, 1984.

DYER, L.; HOLDER,G. Toward a strategic perspective of human resource management. In Dyer (ED), Human resource management: Evolving roles and responsibilities, ASPA/BNA 
Handbook of Human Resource Management. Washington: Bureau of National Affairs, 1988.

FERREIRA, J.F.; TEIXEIRA, M.L.M. Papéis de Recursos Humanos: Uma Visão Ampliada pelas Ações e Expectativas Percebidas. Sociedade, Contabilidade e Gestão, 1(1), 2006.

FREITAG, B.B.; GIRARDI, D. Consultoria interna de $\mathrm{RH}$ em um empresa de pequeno porte. EnANPAD, 2009.

EVANS, W. The Organization Set: Toward a Theory of Inter-organizational Relations. In J. Thompson(Ed), Approaches in organization design, 173-191. Pittsburgh, PA: University of Pittsburgh Press, 1996.

FRIEDMAN, M. The Social Responsibility of Business is to Increase its Profits. Springer Berlin Heidelberg, 2007.

GUEST, D. Human resource management and industrial relations. Journal of Management Studies, 24(5), 503-521, 1987.

GLOBAL HUMAN CAPITAL SURVEY. Executive brief . PriceWaterHouseCoopers, 2002.

HARRIS, P. R.; MORGAN, R. T. Managing cultural difference (4th ed.). Houston: Houston Gulf Publishing Company, 1996.

HSM MANAGEMENT, 55, mar-abr, 2006.

JOHRI, H. P.; COOPER, J. C.; PROKOPENKO, J. Managing internal consulting organizations: a new paradigm. Advanced Management Journal, 63(4), p. 4-10, 1998.

KENTON, B.; MOODY, D. The Role of the Internal Consultant. Roffey Park Institute. 2003.

LAWLWER III, E.E.; MOHRMAN, S.A. HR as a Strategic Partner: What does it take to make it happen? In CEO Publication, G03-2, p.430, 2003.

LAWLWER III, E.E.; MOHRMAN, S.A. Beyond the vision: What makes HR effective? CEO publication, 2000.

LEGGE, K. Power, innovation and problem solving in personnel management. London, UK: McGraw-Hill, 2005.

LENGNICK-HALL, C.A; LENGNICK-HALL, M.L. Interactive Human Resource Management and Strategic Planning. In Westport, CT: Quorum Books, 1990.

LEMMERGAARD, J. (2009). From administrative expert to strategic partner. In Employee Relations, 31 (2), 182-196, 2009.

MABEY, C., SKINNER, D., CLARK, T. Experiencing Human Resource Management. London: Sage Publications Inc, 1998.

MANCIA, L.T.S. Os desafios do modelo de consultoria interna: uma experiência gaúcha. Porto Alegre : Universidade Federal do Rio Grande do Sul/EA/PPGA, 1997.

MOURA, A.L.; SOUZA, B.C. Analisando o impacto da consultoria interna no setor public. Revista Economia \& Gestão da PUC Minas, 8(18), 2008.

NADARAJAH, S.; KADIRESAN, V.; KUMAR, R.; KAMIL, N.N.; YOSOFF, Y.M. The Relationship of HR Practices and Job Performance of Academicians towards Career Development in Malaysian Private Higher Institutions. International Conference on Asia Pacific Business Innovation and Technology Management, 57(9), 2012.

KESLER, G. A Model and Process for Redesigning the HRM Role, Competencies and Work in a Major MultiNational Corporation. In HRM Journal, summer 1995.

KNIGHT, K. A Descriptive Model of the IntraFirm Innovation Process. In Journal of Business, 40; 478-96, 1967.

OLIVEIRA, D. P. R. Manual de consultoria empresarial: conceitos, metodologia, práticas. 2. ed. São Paulo: Atlas, 1999. 
PAROLIN, S.R.H; ALBUQUERQUE, L.G. Innovation-oriented strategic management of people: frimesa cooperativa central case. In Rev. Adm. UFSM, Santa Maria, v. 4, n.1, p. 105124, jan./abr. 2011.

PURCELL, J. Best practice or best fit: chimera or cul-de-sac. In Human Resource Management Journal, Vol. 9 No. 3, pp. 26-41, 1999.

REILLY, P. HR shared service and the realignment of HR. IES Report, p. 368, 2000.

SCHULER, R. S. Repositioning the human resource function: Transformation or demise? In Academy of Management Executive, 4(3), 49-60, 1990.

SHRM WorkPlace Forecast: The to Workplace Trends According to HR Professionals. Society for Human Resource Management, 2011

STOREY, J. Developments in the management of Human Resources. Oxford: Blackwell Publishing, 1992.

SOM, A. What drives adoption of innovative SHRM practices in Indian organizations? In The International Journal of Human Resource Management, 18:5, 808-828, 2007.

SUMELIUS, J., SMALE, A., BJORKMA, I. The strategic role of HR in MNC subsidiaries in China between 1999 and 2006. In Chinese Management Studies, 3 (4), 295-312, 2009.

TANNENBAUM, S.I.; DUPUREE-BRUNO, L.M. The Relationship between Organizational and Environmental Factors and the Use of Innovative Human Resource Practices, In Group and Organization Management, 19(2): 171-202, 1994.

TRACEY, J.B.; NATHAN, A.E. The Strategic and Operational Roles of Human Resources: An Emerging Model. Cornell Hotel and Restaurant Administration Quarterly, 43: 17-26, 2002.

TICHY, N. M., FROMBRUM, C.J., DEVANNA, M.A. Strategic Human Resource Management.
Sloan Management Review, 23(2), 47-61, 1982.

TRUSS, C.; GRATTON, L.; HOPE-HAILEY, V.; McGOVERN, P.; STILES, P. Soft an Hard Models of Human Resource Management: A Reappraisal. In Journal of Management Studies, 34:1, 1997.

ULRICH, D. Measuring Human Resources: An Overview of Practice and a Prescription for Results, In Human Resource Management, 36(3): 303-20, 1997.

ULRICH, D.; YOUNGER, J.; BROCKBANK, W. The Twenty-first Century HR Organization. In Human Resource Management, vol.47, (4):829-850, 2008.

ULRICH, D. Os Campeões de Recursos Humanos. São Paulo: Editora Futura, 2001.

WANG, Y. D.; NIU, H. J. Multiple roles of human resource department in building organizational competitiveness-Perspective of role theory. In International Management Review, 6 (2), 13-19, 2010.

ULRICH, D., BROCHBANK, W. The HR Value Proposition. Boston: Harvard Business School Press, 2005.

VALOR ECONÔMICO, 2012. http://www.valor. com.br/carreira/2547216

VOCÊSA. http://exame.abril.com.br/noticia/ business-partner-longe-do-mundo-ideal/ imprimir

VOSBURGH, R.M. The Evolution of HR: Developing HR as an Internal Consulting Organization. Human Resource Planning, 30(3), p11-23, 2007.

WERS Inside the Workplace.Workplace Employment Relations Survey, 2004.

WOLFE, R.A. Human Resource Management Innovations: Determinants of Their Adoption and Implementation, In Human Resource Management, 34(2): 313-27, 1995. 
WILEY, C. A comprehensive view of roles for human resource managers in industry today. In Industrial Management, 34(6), 27-29, 1992.

WILHELM, W. Revitalizing the Human Resource Management Function in a Mature, Large Corporation, In Human Resources Management, 29(2), 129-144, 1990

WOLFE, R.; WRIGHT, P.M.; SMART, D.L. Radical HRM Innovation and Competitive Advantage: The Moneyball Story. In Human Resource Management, Vol. 45, No. 1, Pp. 111-145, 2006.

WRIGHT, C. Reinventing human resource management: Business partners, internal consultantsand the limitstoprofessionalization. In Human Relations, 2008.

WRIGHT, P.M., SNELL, S.A. Toward a uniflying framework for exploring fit and flexibility in strategic human resource management. Academy of Management Review, 23(4), 756772, 1998. 